\title{
Bessel-Beam Hyperspectral CARS Microscopy with Sparse Sampling: Enabling High-Content High-Throughput Label-Free Quantitative Chemical Imaging
}

\author{
Francesco Masia, $^{\dagger, \S}$ Iestyn Pope, ${ }^{\ddagger}{ }^{\ddagger}$ Peter Watson, ${ }^{\ddagger}$ Wolfgang Langbein, ${ }^{\dagger}$ and Paola Borri* ${ }^{*}{ }^{\ddagger}$ \\ 'School of Physics and Astronomy, Cardiff University, The Parade, Cardiff CF24 3AA, U.K. \\ ${ }^{\ddagger}$ School of Biosciences, Cardiff University, Museum Avenue, Cardiff CF10 3AX, U.K.
}

Supporting Information

\begin{abstract}
Microscopy-based high-content and highthroughput analysis of cellular systems plays a central role in drug discovery. However, for contrast and specificity, the majority of assays require a fluorescent readout which always comes with the risk of alteration of the true biological conditions. In this work, we demonstrate a label-free imaging platform which combines chemically specific hyperspectral coherent anti-Stokes Raman scattering microscopy with sparse sampling and Bessel beam illumination. This enabled us to screen multiwell plates at high speed, while retaining the high-

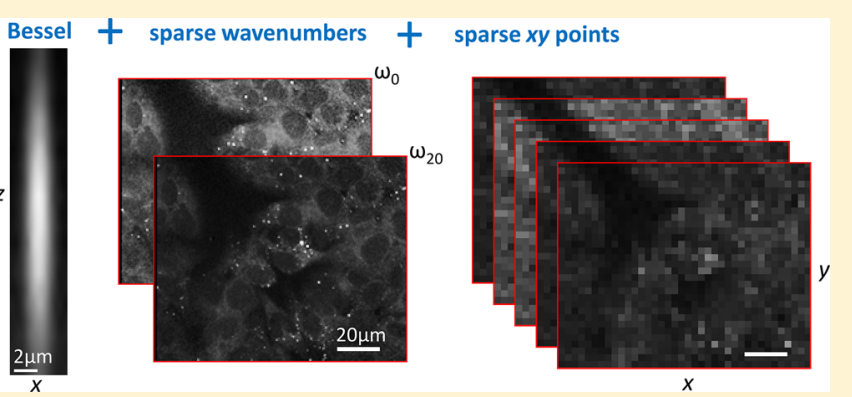
content chemical analysis of hyperspectral imaging. To demonstrate the practical applicability of the method we addressed a critical side effect in drug screens, namely, drug-induced lipid storage within hepatic tissue. We screened 15 combinations of drugs and neutral lipids added to human HepG2 liver cells and developed a high-content quantitative data analysis pipeline which extracted the spectra and spatial distributions of lipid and protein components. We then used their combination to train a support vector machine discriminative algorithm. Classification of the drug responses in terms of phospholipidosis versus steatosis was achieved in a completely label-free assay.
\end{abstract}

$\mathrm{F}$ or many years high-throughput high-content microscopy has played a central role in the discovery and development of therapeutics through the screening of phenotypes. ${ }^{1}$ Imaging screens are not only able to define positive phenotypes but also indicate potential unwanted side-effects. To develop contrast within the specimen, the majority of high-content assays require modification of the biological system with a fluorescent readout. ${ }^{2,3}$ While inclusion of a fluorescent reporter is a powerful technique, it precludes the use of unmodified primary tissues and cell types and always incorporates the risk of alteration of the true biological conditions of the system under test. In addition, multiplexing of fluorescent readouts is typically limited to a small number that can be well separated by optical filters. ${ }^{3}$

An example of a critical side effect that needs to be assessed in drug screens and is specifically hard to detect in current fluorescent-based assays is drug-induced lipid storage within hepatic tissue. The liver plays a central role in the detoxification of foreign molecules and drugs and, thus, is particularly susceptible to drug induced injury. The onset of toxicity within hepatic tissue has led to the cancelation of clinical trials, and is the greatest cause of the withdrawal of commercially available therapeutics following approval. ${ }^{4,5}$ Drug-induced liver injury (DILI) includes steatosis and phospholipidosis, which involve the production of cellular structures containing excess lipid. Drug-induced steatosis (DIS) is the accumulation of lipids in the form of diacyl and triacylglycerols within intracellular lipid droplets with a phospholipid monolayer surrounding the lipid core, and is often caused by an off-target effect of the drug that results in mitochondrial damage. ${ }^{6}$ Phospholipidosis is the accumulation of phospholipid membranes within a multilayered/multilamellar structure. Because of the potential for drug induced lipid storage to (a) prevent the development and progress of drugs into the clinic and (b) to lead to the development of potentially life threatening side effects once administered, it is important to not only detect the onset of these problems as early as possible within the development cycle but also clearly define which lipid storage phenotype is prevalent.

End point assays are available for both phospholipidosis and steatosis; however, the addition of fluorescent components has major limitations since lipid-binding dyes photobleach severely and may affect development. The gold standard of defining phospholipidosis is the use of electron microscopy to identify lipid rich cellular structures with a morphology resembling an onion, which is laborious and time-consuming. What is required is a label-free noninvasive method that is capable of detecting the presence of, and the difference between phospholipidosis and steatosis, yet is fast and amenable to

Received: October 2, 2017

Accepted: March 5, 2018

Published: March 5, 2018 


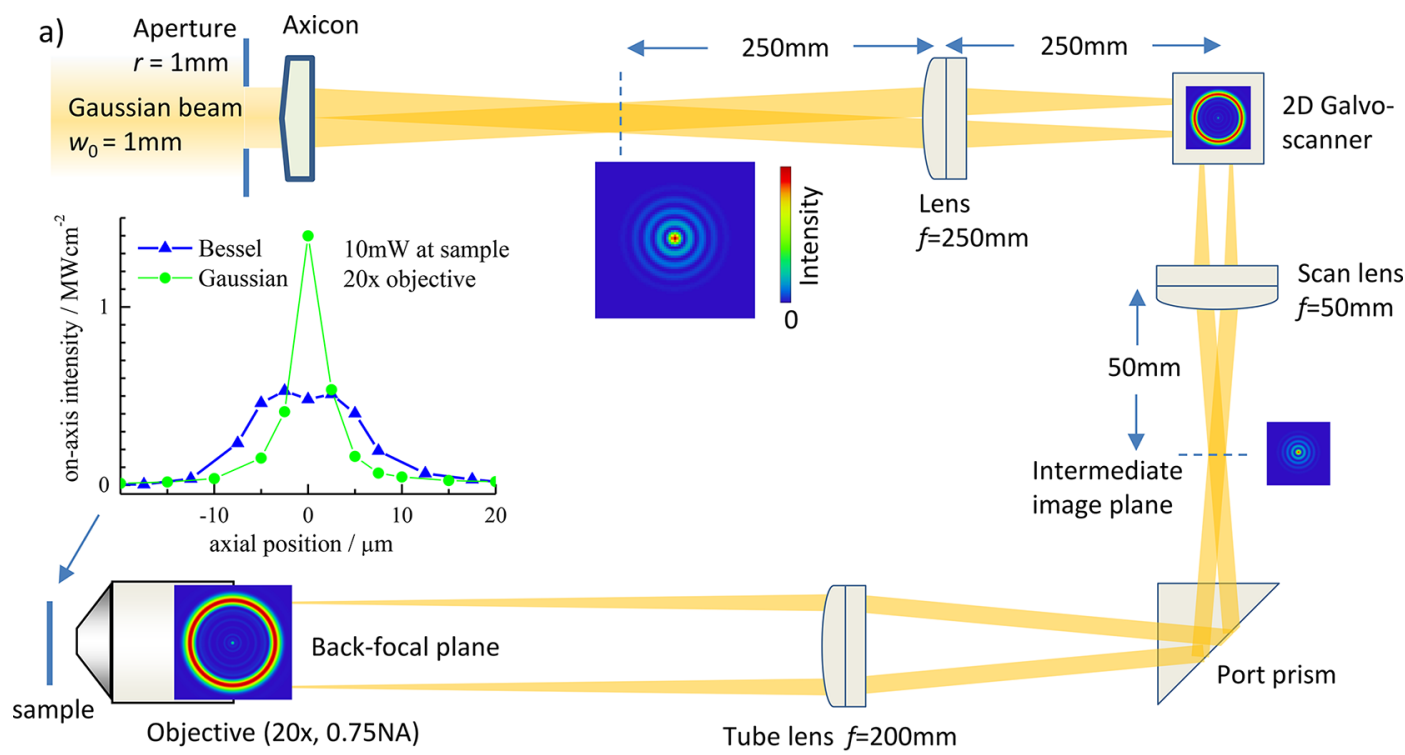

b)

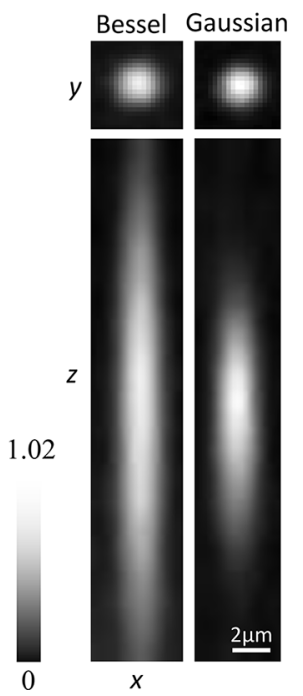

c)
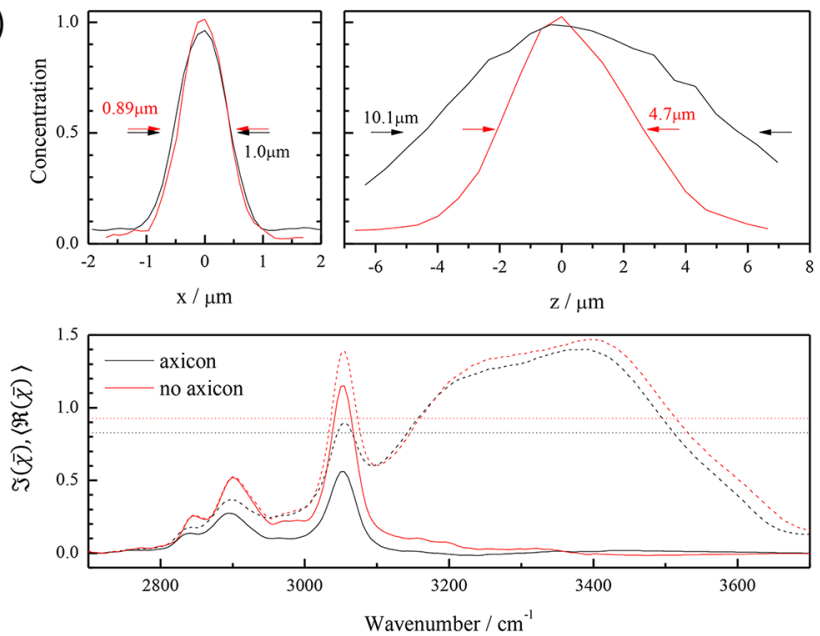

Figure 1. (a) Sketch of the optical setup. To switch between Gaussian and Bessel beam excitation, an aperture, an axicon, and a lens are inserted into the parallel beam path in front of the galvo scanner, prior to entering the optics (scan lens, prism, tube lens, objective) of a commercial inverted microscope. The axicon with about $4 \mathrm{mrad}$ deflection angle generates a finite Bessel-beam of $250 \mathrm{~mm}$ extension for a $1 \mathrm{~mm}$ radius input beam determined by the aperture. The resulting lateral intensity distributions are shown in the central plane of the Bessel beam after the axicon, at the galvo-scanner, in the intermediate image plane, and at the objective back focal plane, on a color scale as given. The graph shows the axial intensity distribution, for $10 \mathrm{~mW}$ beam power at the sample. b,c) Comparison between hyperspectral CARS imaging acquired with Bessel and Gaussian beam excitation on a polystyrene bead of $1 \mu \mathrm{m}$ nominal size in agar. Retrieved concentration maps ( $x y$ focal plane and $x z$ equatorial-axial) of polystyrene ( $\mathrm{vol} / \mathrm{vol}$ ) are shown in panel b. Corresponding line cuts and spectral profiles of the CARS susceptibility imaginary part (dashed lines) and spectrally averaged real part (dotted lines) are shown in panel c. Solid lines show the spectra after subtracting the water component. Acquisition settings: 0.122 $\mu \mathrm{m}$ lateral pixel size, 21 axial steps with $0.66 \mu \mathrm{m}$ step size, $5 \mathrm{~cm}^{-1}$ wavenumber step size, $0.1 \mathrm{~ms}$ pixel dwell time. Dry objective $20 \times 0.75 \mathrm{NA}, 1 \times$ tube lens. Pump (Stokes) power at the sample was $58 \mathrm{~mW}(33 \mathrm{~mW})$ for Bessel beam excitation and $11 \mathrm{~mW}(7 \mathrm{~mW})$ for Gaussian beam excitation.

high-throughput methods, ideally applicable to living cells so that the progress of drug induced effects can be monitored over time. Importantly, the development of a readout that can track the progress of DILI would also provide a tool allowing the screening of therapeutic treatments of DIS, which is a current unmet need within the field. ${ }^{7}$

Coherent Raman scattering (CRS) microscopy has emerged in the last two decades as a powerful label-free chemically specific multiphoton microscopy technique. ${ }^{8}$ In Raman scattering, the interaction of light with vibrating chemical bonds gives rise to light at a different wavelength compared to the incident field. The wavelength shift is a direct signature of the frequency of the vibration which in turn depends on the type of chemical bond. This scattering phenomenon usually produces a very weak signal, but can be strongly enhanced in CRS when two short laser pulses (denoted as pump and Stokes of optical frequencies $\omega_{\mathrm{p}}$ and $\omega_{\mathrm{s}}$ respectively) are used to coherently drive the vibrations at frequency $\omega_{\mathrm{p}}-\omega_{\mathrm{s}}$, such that Raman scattered light from coherently driven molecular vibrations in the focal volume constructively interferes.

Chemically specific label-free CRS imaging of living cells with high spatial and temporal resolution has been demonstrated on many biological samples, with particular emphasis on imaging lipids owing to the large number of identical $\mathrm{CH}$ bonds in their 
acyl chains. ${ }^{8,9}$ Initial demonstrations of CRS microscopy focused on showing high-speed imaging up to video rate but with limited chemical information provided solely from detecting at a single $\mathrm{CH}$-stretch vibrational resonance and unable to give a quantitative chemical map of the lipid concentration. ${ }^{10,11}$ Subsequent implementations of multiplex or hyperspectral CRS imaging, where a vibrational spectrum is measured for each spatial point, enabled much higher specificity $^{12}$ and quantitative spatiotemporal chemical profiling. ${ }^{13}$ Notably, substantial progress has been made in the quantitative data analysis associated with hyperspectral imaging when using coherent anti-Stokes Raman scattering (CARS) acquisition. The ability to measure the vibrationally nonresonant part of the third order susceptibility in CARS enables straightforward in situ calibration via ratiometric comparison with a standard nonresonant medium in the sample (e.g., water or the glass coverslip), which in turn results in the quantitative retrieval of susceptibility spectra factorized into several chemical components with physically meaningful units. ${ }^{13-15}$

CARS hyperspectral microscopy appears, therefore, as a promising tool for quantitative high-content noninvasive imaging screens, especially in relation to drug induced lipid storage, however its implementation in a high-throughput multiwell format has not yet been demonstrated. In this work, we have developed a novel CARS imaging platform which combines a Bessel beam illumination, to extend the axial depth of field, with a hyperspectral sparse sampling approach. This enables screening multiwell plates at high speed (here a 45 -fold improvement compared to Gaussian beam illumination and full spectral and spatial acquisition) while retaining the highcontent chemical analysis of hyperspectral imaging. To demonstrate the practical applicability of the method, we screened 15 combinations of drugs and neutral lipids added to human HepG2 liver cells. By combining the spatial and spectral information on the chemical components extracted from the quantitative data analysis, we were able to train a support vector machine (SVM) discriminative algorithm and classify the drug responses in terms of neutral lipid accumulation versus phospholipidosis in a completely label-free assay.

\section{MATERIALS AND METHODS}

Microscope Setup. The microscope was built around an inverted Nikon Ti-U stand as described in ref 16. To switch between Gaussian and Bessel beam excitation, an aperture, an axicon, and a lens were inserted into the parallel input beam path in front of the galvo scanner, as shown in Figure 1, mounted on two flip mirrors for fast and simple exchange from Bessel to Gaussian beams. The axicon is made of fused silica with a tip angle of $179^{\circ}$ (Thorlabs AX2505-B) and generates a conical wavefront with about $4 \mathrm{mrad}$ deflection angle, providing a finite Bessel-beam of $250 \mathrm{~mm}$ extension for a $1 \mathrm{~mm}$ radius input beam determined by the added input aperture (Thorlabs SM1D12) matching the Gaussian beam waist $w_{0}=1 \mathrm{~mm}$ of the input beam. Placing the axicon with the tip toward the laser source avoids the direct back-reflection of the collimated beam by the flat surface, which could affect laser operation. An achromatic lens (Thorlabs AC254-250-B) with $f=250 \mathrm{~mm}$ focal length is placed at $250 \mathrm{~mm}$ distance from the central plane of the Bessel beam, to image the central plane to infinity. The lens has a distance of $250 \mathrm{~mm}$ from the far-field plane at the galvo scanner, thus imaging the directional space of the Bessel beam, shown as lateral intensity distribution in Figure 1, into the far-field plane conjugated to the objective back focal plane.
For the chosen elements the ring radius is about $1 \mathrm{~mm}$, similar to the Gaussian beam and thus compatible with a simple switch between both beams using the same magnification to the objective back focal plane. The scan lens (Nikon) of $f=50 \mathrm{~mm}$ then images the Bessel beam center into the intermediate image plane, from where it is imaged by the $1 \times$ tube lens of focal length $f=200 \mathrm{~mm}$ and the $20 \times 0.75$ NA objective of $f=10 \mathrm{~mm}$ into the sample. Furthermore, since the fill factor for the Gaussian beam is only 0.55 with the $1 \times$ tube lens, by changing the tube lens to $1.5 \times$ we can decrease the axial depth by a factor $(1.5)^{2}$ and reduce the lateral PSF width by 1.5 , for both Gaussian and Bessel beams.

In the present work, we do not resolve the CARS signal directionally and use a wide collection angle (0.72 NA Condenser). This minimizes the effects of phase matching in our data. Hyperspectral CARS images were acquired as described in ref. ${ }^{13,16,17}$ Briefly, we split the broadband beam (660-970 nm) from a 5 fs Ti:sapphire laser (Venteon Pulse One $\mathrm{PE}$ ) with $80 \mathrm{MHz}$ repetition rate into the pump beam $(660-730 \mathrm{~nm})$ and the Stokes beam $(730-900 \mathrm{~nm})$ for CARS excitation. We use spectral focusing in which an equal linear chirp is applied to both the pump and Stokes beams, resulting in a constant instantaneous frequency difference (IFD), which can be tuned by varying the delay between pump and Stokes beams. The resulting pulse duration at the sample is about 1.5 ps, corresponding to an IFD resolution of about $10 \mathrm{~cm}^{-1}$. The CARS signal is collected by the condenser, separated from the excitation beams using two Semrock FF01-562/40 filters and detected by a photomultiplier (Hamamatsu H7422-40). Data were typically acquired in the wavenumber range 2600 to 3800 $\mathrm{cm}^{-1}$. Specific settings for each measurements are indicated in the captions. Note that our sequential hyperspectral CARS acquisition with spectral focusing has a typical time per spectrum of $\sim 2 \mathrm{~ms}$, given by the time per pixel $(10 \mu \mathrm{s})$, and number of spectral point in a spectrum ( 200), which is comparable to the broadband CARS approaches using a spectrometer and a CCD camera reported in the literature. ${ }^{18}$

The microscope stand was also equipped with wide-field differential interference contrast (DIC) optics and epifluorescence illumination and detection, for correlative microscopy. DIC imaging was performed using a de-Senarmont compensator with polarizer angle at 12.5 degrees (i.e., a phase offset of 25 degrees). Fluorescence was performed with a metal-halide lamp at 25\% (Lumen L200/D; Prior Scientific, Rockland, MA) and an exciter/emitter/dichroic filter cube GFP-A-Basic (Semrock, Rochester, NY) for the green channel, and mCherry-40LP-A (Semrock, Rochester, NY) for the red channel. All images were acquired at $100 \mathrm{~ms}$ frame exposure, except for fluorescence in the green channel which was acquired at $1 \mathrm{~s}$ frame exposure.

Cell Culture. All cell media reagents were purchased from Invitrogen Life Technologies/ThermoFisher Scientific (Waltham, MA). Oleic acid and phospholipidosis inducers were purchased from Sigma (St. Louis, MO). HeLa cells (ATCC CCL-2) were grown on coverslips or microwell slides (Hamamatsu A10657-01) in DMEM supplemented with 10\% (v/v) FCS and $2 \mathrm{mM}$ L-glutamine. Fluorescent detection of phospholipidosis was achieved using LipidTOX Red phospholipid stain according to the manufacturer's instructions. Briefly, a $2 \times$ stock of phospholipidosis reagent is prepared in growth media, filter sterilized, and added to the coverslips in a 6 well tissue culture dish. To the appropriate wells, an equivalent volume of growth media containing $2 \times$ test compounds is added. Cells were then incubated for $72 \mathrm{~h}$ under standard tissue 


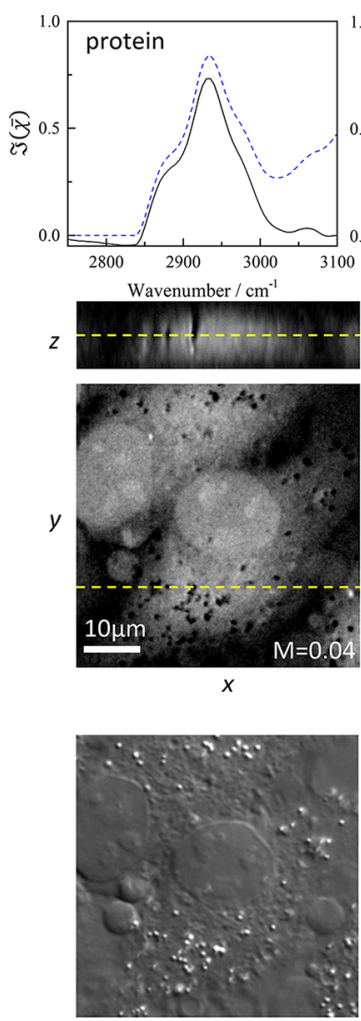

DIC

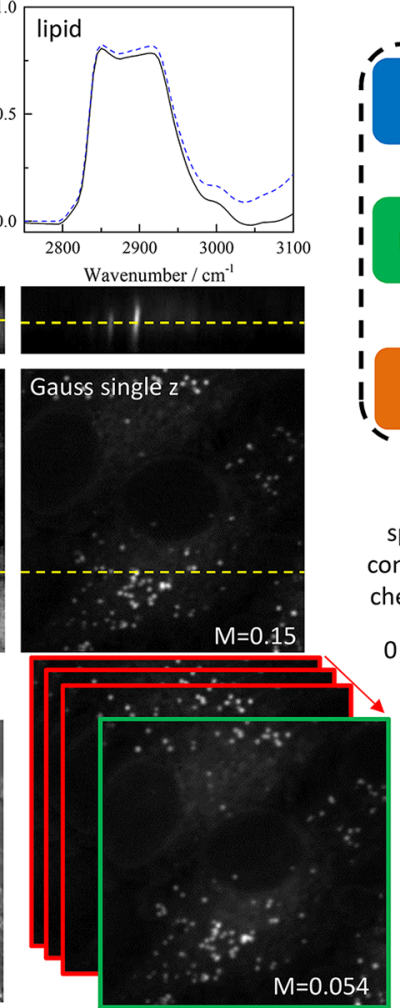

Gauss z-stack weighted average unsupervised

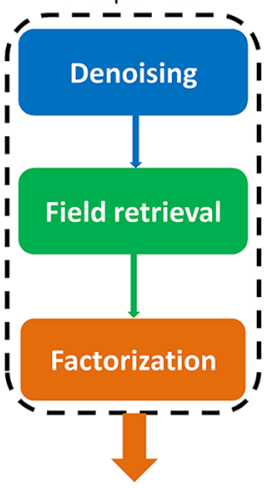

spectra and vol:vol concentration maps of chemical components

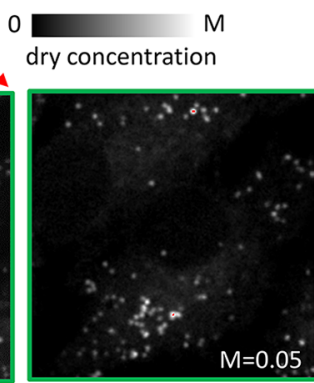

Bessel single $z$

Figure 2. Quantitative unsupervised retrieval of chemical components from label-free hyperspectral CARS in HeLa cells; comparison between Gaussian and Bessel beam. Top right: Sketch of the quantitative data analysis pipeline, comprising denoising, field retrieval, and factorization of susceptibility spectra into independently varying chemical components and their volume concentrations. Top left: Spectra and concentration maps of the dry components corresponding to proteins and lipids. Dashed lines are spectral profiles of the CARS susceptibility imaginary part. Solid lines show the spectra after subtracting the water component. Gray scales are vol/vol concentrations between 0 (black) and M (white) indicated for each panel. The top four images are the $x z$ and $x y$ concentration maps obtained by imaging the cells with Gaussian beam excitation. The yellow dashed lines indicate the position of the cuts. The weighted average 2D projection of these 3D data is shown for the lipid component in the bottom (center), and compared with a single in-plane image acquired with Bessel beam excitation (bottom right). Bottom left shows a differential interference contrast image (DIC) of the same region. Acquisition settings: $0.175 \mu \mathrm{m}$ lateral pixel size, $5 \mathrm{~cm}^{-1}$ step size, $0.01 \mathrm{~ms}$ pixel dwell time, for Gaussian beam excitation 9 axial steps with $1.3 \mu \mathrm{m}$ step size. $1 \times$ tube lens. Pump (Stokes) power onto the sample was $44 \mathrm{~mW}(24 \mathrm{~mW})$ for Gaussian beam excitation and $99 \mathrm{~mW}(50 \mathrm{~mW})$ for Bessel beam excitation. Time per hyperspectral 2D image (241 spectral points) is $196 \mathrm{~s}$.

culture conditions. Following the incubation, cells were fixed with $4 \%$ paraformaldehyde in PBS for $20 \mathrm{~min}$, and then washed with PBS. Fluorescent detection of neutral lipid droplets was achieved using LipidTOX Green neutral lipid stain according to the manufacturer's instructions. Briefly, following PFA fixation coverslips containing cells were incubated in $1 \times$ LipidTOX green neutral lipid detection solution diluted in PBS for $20 \mathrm{~min}$, followed by 3 washes in fresh PBS. Coverslips were then mounted using adhesive imaging spacers (Grace BioLaboratories) and PBS on microscope slides. Microwell slides were maintained in sterile PBS. HepG2 (ATCC HB-8065) cells were seeded at 2500 cells in each well of a Microwell slide (Hamamatsu A10657-01) in DMEM supplemented with 10\% (v/v) FCS and $2 \mathrm{mM}$ L-glutamine. For lipid analysis, $24 \mathrm{~h}$ post seeding cells were incubated overnight with BSA conjugated oleic, linoleic or $\alpha$-linolenic acids at $47 \mu \mathrm{g} / \mathrm{mL}$. For lipidosis/ phospholipidosis screening, $24 \mathrm{~h}$ postseeding cells were incubated overnight in media containing $20 \mu \mathrm{M}$ propranolol, $10 \mu \mathrm{M}$ chlorpromazine, $30 \mu \mathrm{M}$ chloroquine, $10 \mu \mathrm{M}$ tamoxifen, $0.1 \%(\mathrm{v} / \mathrm{v})$ deionized water, $1 \%(\mathrm{v} / \mathrm{v})$ ethanol or full media, with (wells $\mathrm{C} 1-\mathrm{C} 7$ ) or without (A1-A7 and $\mathrm{B} 1-\mathrm{B} 7)$ a supplement of $9.4 \mu \mathrm{g} / \mathrm{mL}$ BSA conjugated Oleic acid (Sigma). Note that the LipidTOX phospholipidosis and steatosis detection kit is the current standard for high-throughput imaging, as shown in ref 19, where all phospholipidosis inducers used in our study were identified with this kit, and confirmed by comparison with electron microscopy.

\section{RESULTS AND DISCUSSION}

Bessel Beam Generation and Characterization. A focused Bessel beam can provide a nonlinear probe volume with an axial extension encompassing the full thickness of a $2 \mathrm{D}$ cell culture in a multiwell plate (i.e., several microns), while retaining high confinement in-plane, thus enabling the sample's lateral organization to be probed at high resolution while averaging axially for fast acquisition.

Bessel beams have been recently proposed as a way to achieve rapid volumetric imaging via extended depth of field in nonlinear microscopy based on two-photon fluorescence, ${ }^{20,21}$ second harmonic generation contrast, ${ }^{22}$ and single-frequency stimulated Raman scattering. ${ }^{23}$ However, their implementation for high-throughput hyperspectral CARS microscopy has not yet been shown. Interestingly, their opposite use was discussed in ref. $^{24,25}$ to retrieve the axial structure of a laterally homogeneous sample from phase matching considerations and the directionality of the CARS emission. 

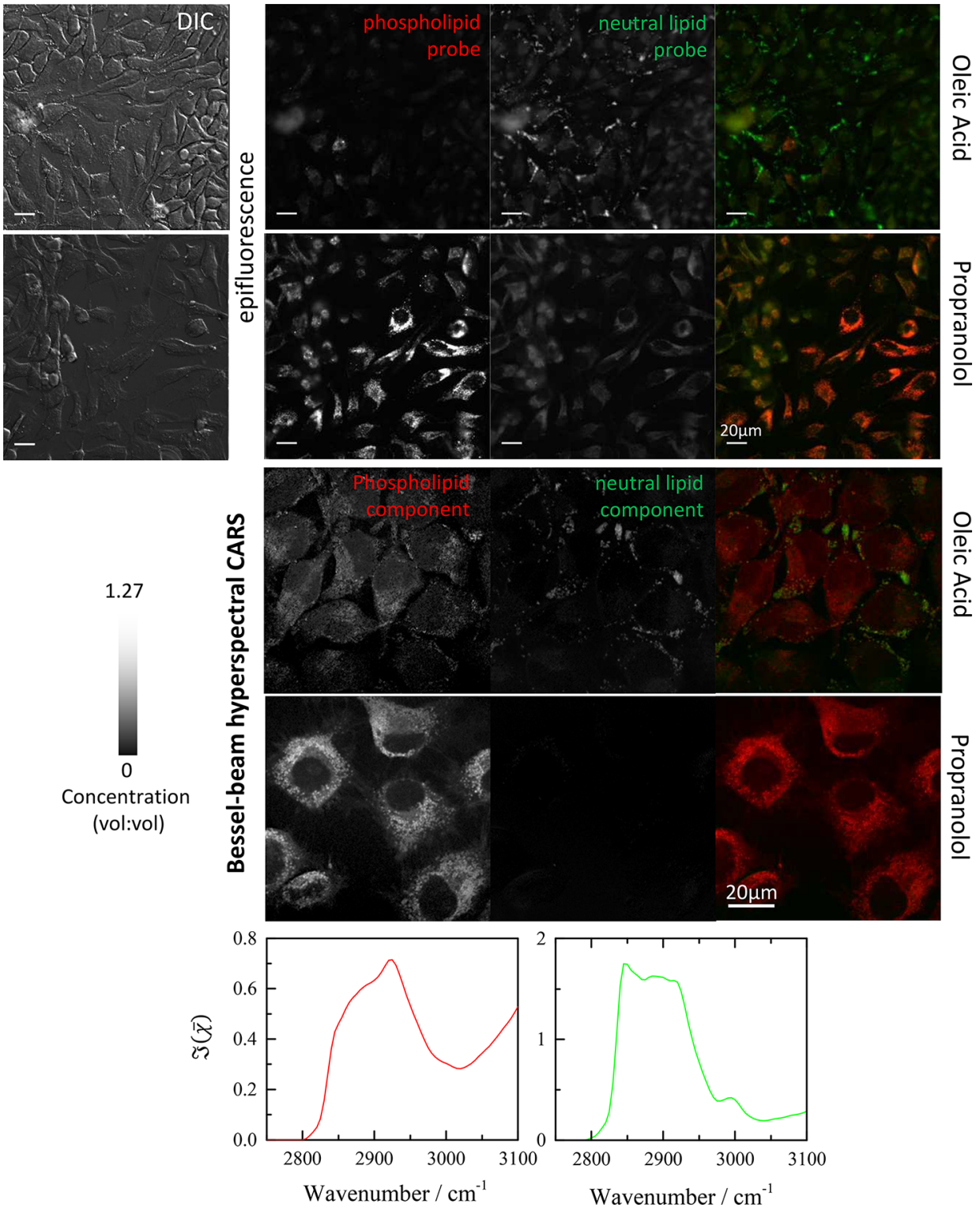

Figure 3. Label-free markers of phospholipidosis and neutral lipid accumulation. HeLa cells grown in media supplemented with oleic acid were compared with HeLa cells exposed to the phospholipidosis inducer propranolol. The top two rows show HeLa cells fluorescently labeled with the phospholipid probe LipidTOX Red (red) and the neutral lipid probe LipidTOX Green (green), imaged by DIC (left) and wide-field epifluorescence. Each fluorescence channel is shown in black and white and as red-green overlay. The bottom rows show nominally identical unstained Hela cells with the concentration maps and spectra of the lipid components retrieved from the analysis of hyperspectral CARS imaging. Concentration maps are given in black and white on the indicated scale, and color coded as red-green overlay to exemplify the similarity with the lipid staining (red: phospholipidosis component, green: neutral lipid components; spectra as indicated). CARS acquisition settings: $0.122 \mu \mathrm{m}$ lateral pixel size, $5 \mathrm{~cm}^{-1}$ step size, $0.01 \mathrm{~ms}$ pixel dwell time. $1.5 \times$ tube lens. Pump (Stokes) power onto the sample was $53 \mathrm{~mW}(27 \mathrm{~mW})$. Time per hyperspectral image (241 spectral points) is $1620 \mathrm{~s}$.

Most experimental implementations reported use a liquid crystal phase shaper array to create the Bessel beam. ${ }^{22}$ This method is very flexible, as Bessel beams of different cone angle can be created, but is also rather lossy (60\% transmission was reported $^{22}$ at $840 \mathrm{~nm}$ ), complex, costly, and requires active control. We have opted here for a simple solution using a single axicon (instead of two in refs 24 and 25) to create the Bessel beam, which allows for high transmission (>90\% over a broad wavelength range of $650-1100 \mathrm{~nm}$ ) and passive stability. Three simple elements (an aperture, an axicon, and a lens) are mounted on flip mounts, so that they can be easily switched in and out of the beam path, allowing access to both the Gaussian and Bessel beam excitation without realignment (see Figure 1a and Methods).

The lateral intensity distribution in the central plane of the Bessel beam after the axicon has the typical Bessel-beam shape with a sharp central maximum surrounded by rings of intensity decaying with the inverse of the radius (see Figures la and S1). The axial intensity distribution at the sample is also shown in Figure 1a. While the Gaussian beam has a short depth of field of about $3.5 \mu \mathrm{m}$, the Bessel beam has an axial extension of about $10 \mu \mathrm{m}$, resulting in an extended depth of field. The addition of the aperture results in a top-hat shape of the intensity, and provides a better defined extension of the Bessel beam (see also Figure S2). This is specifically important for CARS since the 

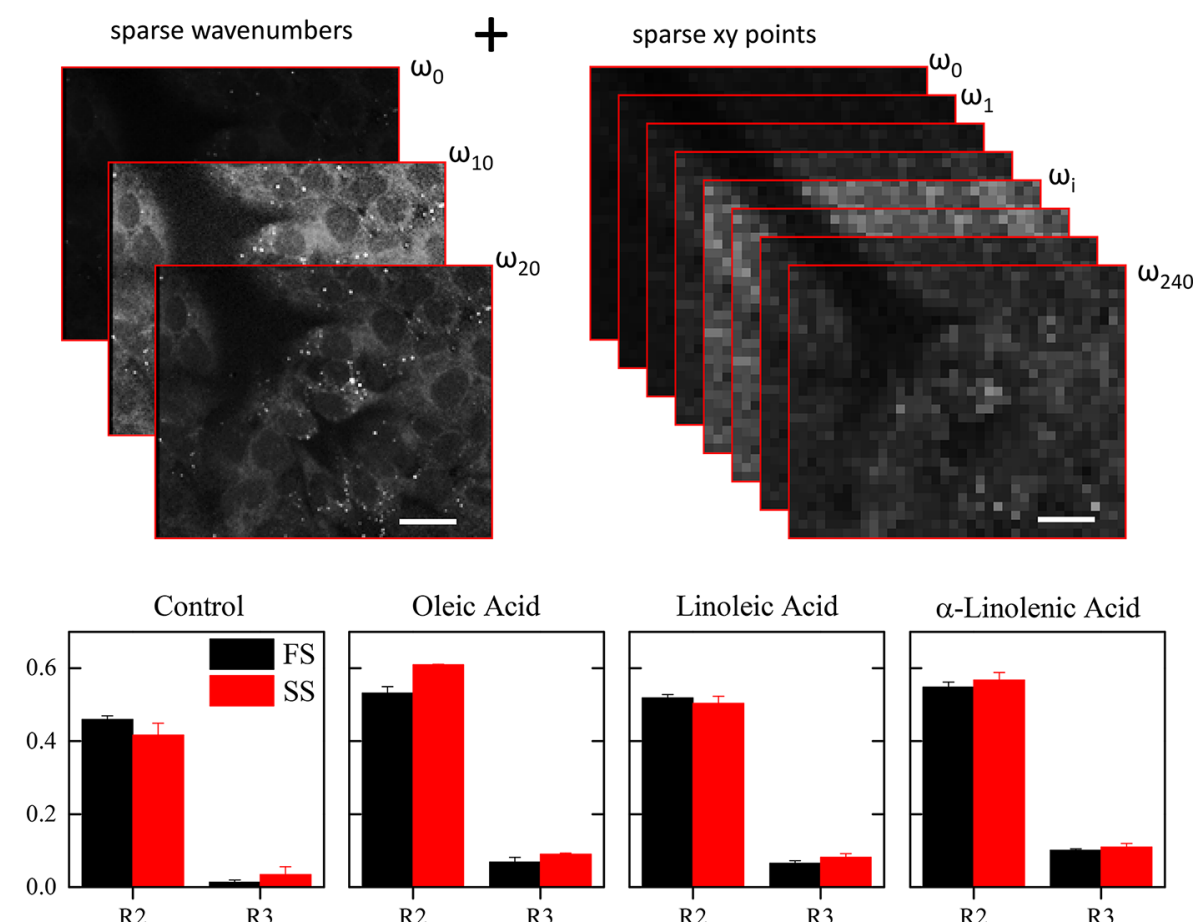

$\alpha$-Linolenic Acid
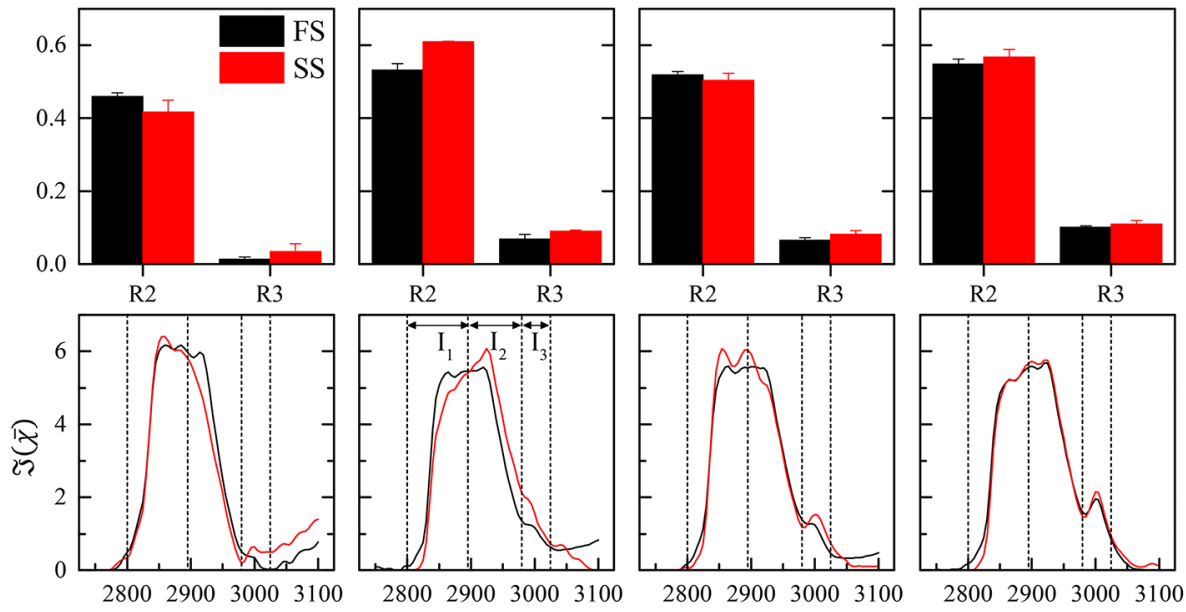

Wavenumber $/ \mathrm{cm}^{-1}$

Wavenumber $/ \mathrm{cm}^{-1}$

Wavenumber $/ \mathrm{cm}^{-1}$

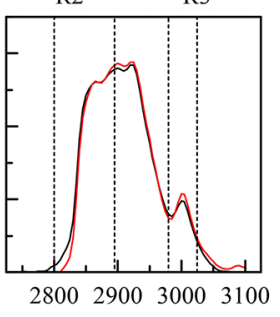

Wavenumber $/ \mathrm{cm}^{-1}$

Figure 4. Sparse sampling with Bessel beam for high speed chemical analysis. Top: Sketch of the Sparse sampling (SS) acquisition combining a CARS image acquired over a small number of spectral points (i.e., vibrational frequencies $\omega_{\mathrm{i}}$ ) and a large number of spatial points (to fully resolve the spatial features of interest), with a hyperspectral image measured on a sparse set of spatial positions over a large number of spectral points (to fully resolve the vibrational resonances of interest). Scale bar: $20 \mu \mathrm{m}$. Bottom: CARS susceptibility spectrum for the lipid component in HepG2 cells grown in media supplemented with monounsaturated oleic acid (OA), biunsaturated linoleic acid and triunsaturated $\alpha$-linolenic acid (red line, SS; black line, Full spectral and spatial (FS)). Ratio of integrated spectra in the regions indicated by the dotted lines are also shown $\left(R_{2}=I_{2} / I_{1}\right.$ and $R_{3}=$ $I_{3} / I_{1}$ ). The error bar indicate the standard deviation obtained by performing 10 repetitions of the $\mathrm{FSC}^{3}$ as an indication of the repeatability of the analysis. FS acquisition settings: $0.122 \mu \mathrm{m}$ lateral pixel size, $5 \mathrm{~cm}^{-1}$ step size. SS acquisition settings: $5 \mathrm{~cm}^{-1}$ step size and pixel size $0.365 \mu \mathrm{m}$ for data with full spectral information; $\sim 30 \mathrm{~cm}^{-1}$ step size and pixel size $0.122 \mu \mathrm{m}$ for data with full spatial information. Number of spectral and spatial points $s=21, S=241, p=274 \times 329, P=820 \times 983$. Pixel dwell time $0.01 \mathrm{~ms} .1 .5 \times$ tube lens. Pump (Stokes) power onto the sample $\sim 30 \mathrm{~mW}(18 \mathrm{~mW})$. Time per image in SS is $(s \times P+p \times S) \mathrm{ms}=386 \mathrm{~s}$, and in FS is $S \times P \times 0.01 \mathrm{~ms}=1942 \mathrm{~s}$.

measured chemical composition is an average material response over the Bessel beam axial extension. Note that for the thickness of the $2 \mathrm{D}$ cell culture investigated by us (see following sections), this axial extension is optimally designed. Further extension of the Bessel beam depth would only add unwanted volume and in turn CARS background.

Figure $1 \mathrm{~b}$ and $\mathrm{c}$ shows the comparison between hyperspectral CARS imaging acquired with Bessel and Gaussian beam excitation on a polystyrene bead of $1 \mu \mathrm{m}$ nominal size in aqueous environment ( $2 \%$ agarose gel). Data were analyzed using our previously developed quantitative data analysis pipeline (called FSC $^{3}$ method). ${ }^{14,15,17}$ This analysis enables us to retrieve the CARS susceptibility spectrum, which is linear in the concentration of chemical components, and factorizes it into independently varying chemical components and their volume concentrations (see sketch in Figure 2 and Supporting Information). Typical vol/vol concentration errors of the retrieval are $1 \%$ absolute and $10 \%$ relative. ${ }^{17}$ Results in Figure
$1 b, c$ are shown as retrieved concentration maps ( $x y$ focal plane and $x z$ equatorial-axial) of the polystyrene component (vol/ vol) with corresponding line cuts, and spectral profiles of the CARS susceptibility. Solid lines show the spectra of 'dry' polystyrene, after subtracting the water component which is present in the focal volume. The concentration maps exemplify the elongated axial distribution, as expected, for Bessel beam excitation, without degradation of the lateral resolution compared to Gaussian beam excitation. The spectral profile of polystyrene measured with Bessel beam exhibits the same vibrational resonances as with Gaussian beam excitation, the only difference being a relatively larger water content in the extended focal volume.

Label-Free Cell Imaging: Bessel Beam versus Gaussian Beam. Figure 2 demonstrates the ability of CARS Bessel beam excitation to provide a chemically resolved map of the lipid distribution in $\mathrm{HeLa}$ cells through a single in-plane acquisition, equivalent to an axially averaged z-stack acquisition 
over 9 planes with a Gaussian beam. Cells were prepared as detailed in the Methods. The same region was imaged with Gaussian beam and with Bessel beam, and the resulting quantitative chemical analysis of the lipid component is shown. As mentioned, data were analyzed using our previously developed data analysis FSC $^{3}$ algorithm, the main steps of which are schematically shown in Figure 2 and described in the Supporting Information. As a result of this analysis, Figure 2 shows the concentration maps and CARS susceptibility spectra of the $\mathrm{FSC}^{3}$ components corresponding to proteins and lipids. The top four images are the $x z$ and $x y$ concentration maps obtained by imaging the cells with Gaussian beam excitation. The weighted average $2 \mathrm{D}$ projection of these $3 \mathrm{D}$ data (using as weight the axial profile of the Bessel beam, see Supporting Information) is shown in the bottom of Figure 2, and compared with a single in-plane image acquired with Bessel beam excitation. The spatial distribution of lipid droplets is equally resolved in both images, demonstrating the ability of Bessel beam excitation to reduce image acquisition without losing information on lateral organization, by $z$-averaging over the entire cell height in a single scan.

Note that the power density used for Bessel beam excitation in Figure 2 is comparable with that used for live cell imaging in ref 13. where we observed human stem cells differentiating into adipocytes over a time course of 9 days, with no apparent damage, arrest or cell death. This is because the peak intensity in the central area for Bessel beam excitation is three times lower than in a Gaussian beam for the same beam power due to the presence of lateral rings in the Bessel beam, as shown in Figure S1.

Label-Free Markers of Phospholipidosis. Using the quantitative unsupervised retrieval of chemical components from hyperspectral CARS imaging in HeLa cells under Bessel beam illumination, we then addressed the capability of the method to derive components able to distinguish phospholipidosis from neutral lipid accumulation. HeLa cells grown in media supplemented with oleic acid (OA) were used as positive control for neutral lipid accumulation and compared with HeLa cells exposed to the drug propranolol, a well-known phospholipidosis inducer. ${ }^{19,26}$ An unsupervised FSC $^{3}$ analysis was performed on cells in propranolol to derive a "phospholipid component" spectrum. Similarly, an unsupervised analysis was performed on cells in OA supplemented media to derive a "neutral lipid component" spectrum. These two spectra were then used as guides in the combined FSC ${ }^{3}$ analysis on both image sets together to finalize the two lipid components (see also Supporting Information). For validation and comparison, nominally identical cells were stained with the red dye LipidTOX Red, which is a marker for phospholipidosis, and the green dye LipidTOX Green neutral lipid stain which associates with neutral lipid droplets, and were imaged with epifluorescence as well as differential interference contrast. The results are summarized in Figure 3. The neutral lipid component correlates well with the green dye staining, markedly present in cells grown in OA supplemented media (notably this component is absent for cells with propranolol). Similarly, the phospholipid component correlates well with the red staining, which is much more pronounced in cells subjected to propranolol. Note that these components were distinct from the protein component which was retrieved as well, in the same combined analysis, and is shown in Figure S3.

Sparse Sampling with Bessel Beam. We recently demonstrated a method based on sparse sampling to increase the acquisition speed in sequential hyperspectral CARS imaging while retaining the relevant spectral and spatial information. ${ }^{14,27}$ Here we show that this method can be utilized together with Bessel beam excitation, to correctly distinguish changes in lipid chemical profiles inside cells, as a prerequisite toward a fast screening platform for drug induced lipid storage. For demonstration, we investigated HepG2 cells grown in media supplemented with either monounsaturated oleic acid, biunsaturated linoleic acid or triunsaturated $\alpha$-linolenic acid (see Methods) to drive systematic changes in the degree of unsaturated neutral lipid content. ${ }^{13,28}$ For comparison, the same cell containing regions were imaged with full spectral and spatial resolution (FS), as well as sparse sampling (SS) acquisition. Typically about 16 cells per sample with a given supplemented medium were imaged and analyzed.

The SS acquisition is briefly sketched in Figure 4 (top). The method consists of combining a CARS image acquired over a small number $(s)$ of spectral points (i.e., vibrational frequencies $\omega_{\mathrm{i}}$ ) and a large number $(P)$ of spatial points (to fully resolve the spatial features of interest), with a hyperspectral image measured on a small number $(p)$ of spatial positions over a large number $(S)$ of spectral points (to fully resolve the vibrational resonances of interest). For the results shown here, the spectral points for sparse sampling acquisition were taken as equidistantly spaced over the full spectral range, which is a simple and close to optimal choice as discussed in ref 27 . A reconstruction algorithm (see Supporting Information) is then applied to generate the full information as if an image with $S$ spectral points and $P$ spatial points was acquired. The method, therefore, reduces the acquisition time to a fraction $(s P+S p) /$ $(S P)=s / S+p / P$.

Data acquired with FS, as well as SS reconstructed data, were then analyzed with our FSC ${ }^{3}$ algorithm (as discussed for Figure 2 and in the Supporting Information). The corresponding CARS susceptibility spectrum for the lipid component is shown in Figure 4 (bottom). The comparison shows that SS retains the relevant spectral information on a FS acquisition, and importantly is able to differentiate between changes in lipid compositions. To further exemplify this point we calculated the integrals of the spectra in the range $2895-2980 \mathrm{~cm}^{-1}\left(I_{2}\right)$ and $2985-3025 \mathrm{~cm}^{-1}\left(I_{3}\right)$ and divided them by the integral of the spectra between 2800 and $2890 \mathrm{~cm}^{-1}\left(I_{1}\right.$; see dotted lines in Figure 2). The corresponding ratios $R_{2}=I_{2} / I_{1}$ and $R_{3}=I_{3} / I_{1}$ are shown in Figure 4 (bottom). The error bar indicates the standard deviation obtained by performing 10 repetitions of the $\mathrm{FSC}^{3}$ as a measure of the repeatability of the analysis (see also Figure S4). The characteristic vibrational bands of neutral lipids include the peak at around $2850 \mathrm{~cm}^{-1}$ which originates from the $\mathrm{CH}_{2}$ symmetric stretch vibrational resonance, the broad shoulder at around $2930 \mathrm{~cm}^{-1}$ which is a combination of $\mathrm{CH}_{3}$ stretch vibrations and $\mathrm{CH}_{2}$ asymmetric stretch enhanced by the broadening and shift of the $\mathrm{CH}$ deformations in the liquid phase, and the $=\mathrm{CH}$ stretch which gives rise to a band around $3010 \mathrm{~cm}^{-1}$. ${ }^{29}$ Therefore, the ratio $R_{2}$ between the band at around $2930 \mathrm{~cm}^{-1}$ and the $\mathrm{CH}_{2}$ symmetric stretch resonance at $2850 \mathrm{~cm}^{-1}$ can be used as a measure of disorder, which usually correlates with the degree of unsaturation. ${ }^{13,28}$ The ratio $R_{3}$ of the $3010 \mathrm{~cm}^{-1}$ peak relative to $2850 \mathrm{~cm}^{-1}$ is a good direct measure of the degree of unsaturation. ${ }^{13,28} \mathrm{We}$ observe that data acquired with SS consistently provide very similar spectral profiles as with FS, and resolve the spectral differences characteristic of an increase in the degree of fatty-acid unsaturation (i.e., increase in $R_{2}$ and $R_{3}$ ) when the cells are 
a) B3 Chlorpromazine
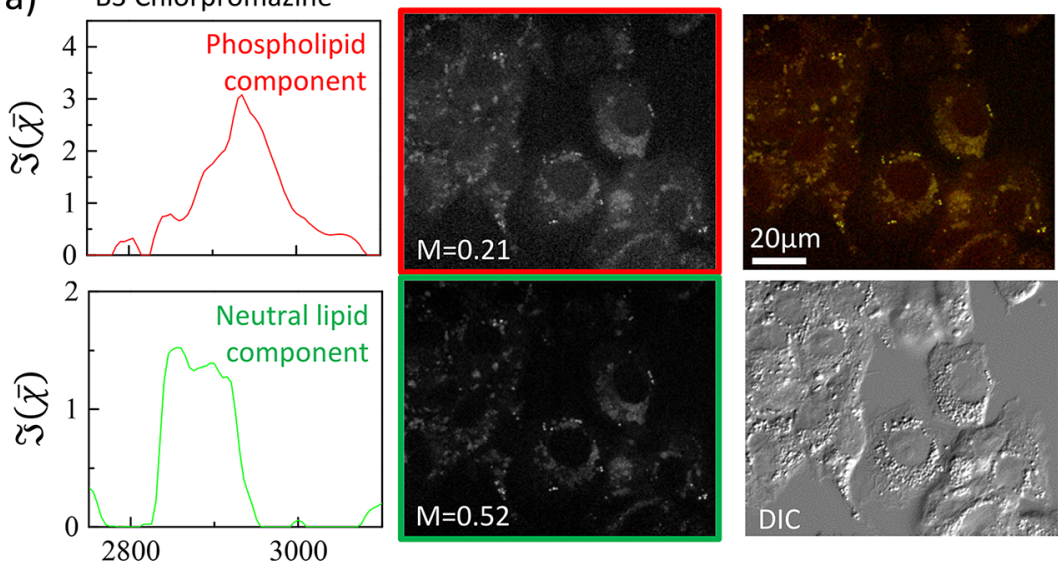

b)

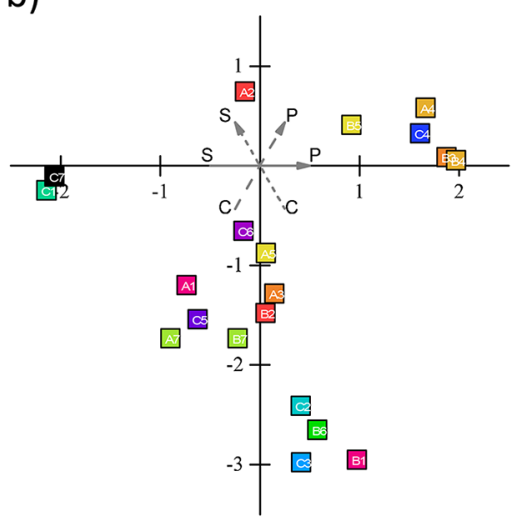

c)

Wavelength / nm
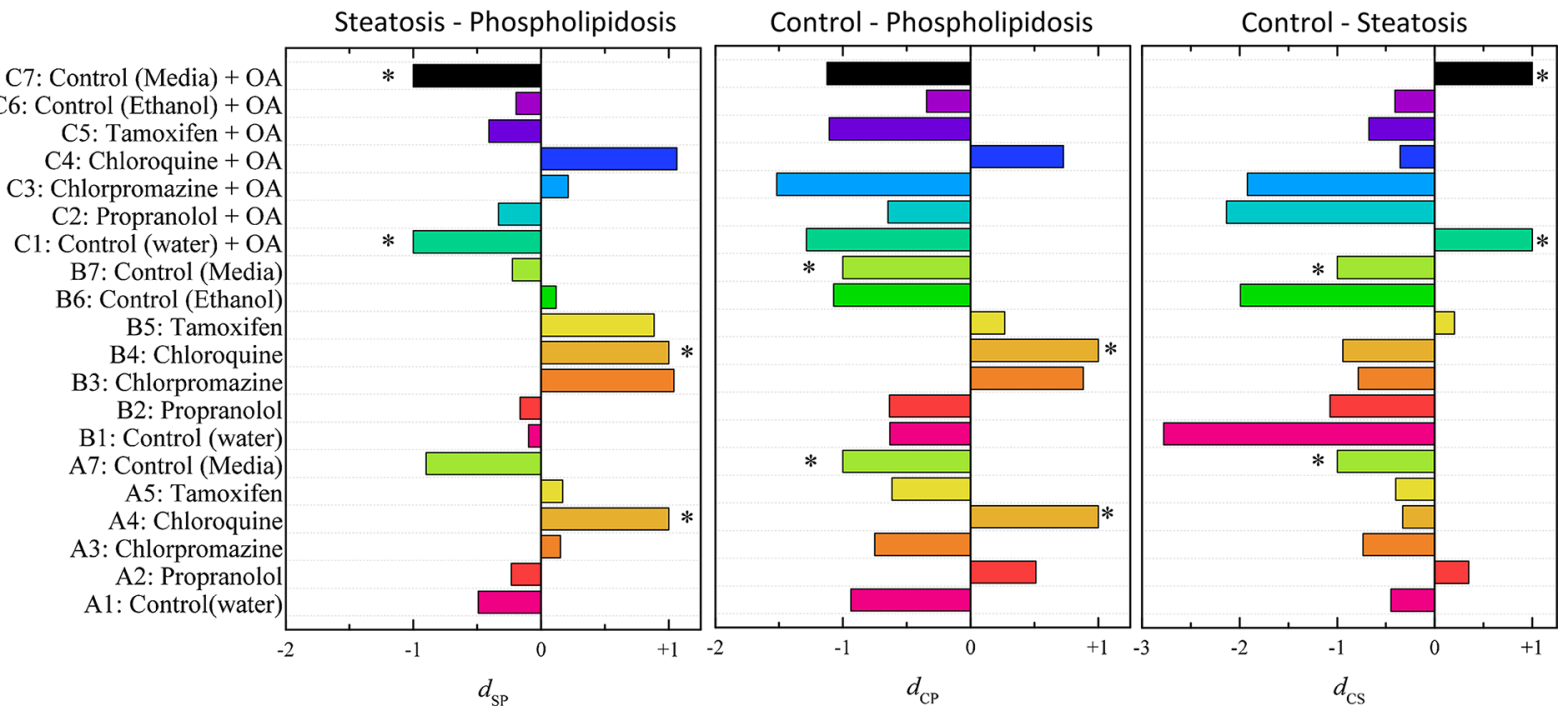

Figure 5. Example of screening for drug-induced lipid storage using hyperspectral CARS with Bessel beam excitation and sparse sampling acquisition. HepG2 cells were treated with various combinations of phospholipidosis and neutral lipid accumulation inducers in a multiwell slide. Panel a shows HepG2 cells in well B3 treated with the phospholipidosis inducer chlorpromazine, imaged by DIC and hyperspectral CARS resulting in factorized lipid spectra and concentration components. Concentration maps are given from 0 (black) to maximum $\mathrm{M}$ (white) vol/vol concentration, as indicated, and as red-green overlay (red, phospholipid component; green, neutral lipid component). Panels b and $\mathrm{c}$ show the outcomes of three binary classifiers using a SVM discriminative algorithm (see text). Panel $c$ gives the results of each binary classification (phospholipidosis (P) versus steatosis (S), phospholipidosis versus control (C), and steatosis versus control), with training sets labeled by the star symbol. Panel b shows the results in a triangular-distance diagram; each well is color coded (as per table in $\mathrm{c}$ ) and is plotted using the distances for each classifier times unit vectors: $(1,0) d_{\mathrm{SP}}+(1 / 2, \sqrt{3} / 2) d_{\mathrm{CP}}+(-1 / 2, \sqrt{3} / 2) d_{\mathrm{CS}}$. The unit vectors are indicated as $(1,0)$ solid, $(1 / 2, \sqrt{3} / 2)$ dashed and $(-1 / 2, \sqrt{3} / 2)$ dotted. SS acquisition settings: $5 \mathrm{~cm}^{-1}$ step size and pixel size $0.365 \mu \mathrm{m}$ for data with full spectral information; $60 \mathrm{~cm}{ }^{-1}$ step size and pixel size $0.122 \mu \mathrm{m}$ for data with full spatial information; number of spectral and spatial points $s=21, S=241, p=274 \times 329, P=820$ $\times$ 983. Pixel dwell time $0.01 \mathrm{~ms}$. $1.5 \times$ tube lens. Pump (Stokes) power onto the sample $37 \mathrm{~mW}(22 \mathrm{~mW})$. Time per image in SS is $386 \mathrm{~s}$.

grown in media supplemented with unsaturated fatty acids compared to standard medium (control), with $R_{3}$ showing a systematic increase when going from monounsaturated (OA) to triunsaturated ( $\alpha$-linolenic) fatty-acid supplement. Spectral cross-correlations of these lipid components are shown in the Table S1. We find that, for the same component, the spectrum acquired with SS differs from the one acquired with FS by less than $0.75 \%$ on average. Notably, differences between lipid components are small in the cross-correlations (2\% to $3 \%$, see Table S1) as these provide an integrated spectral information. The analysis of the ratios $R_{2}$ and $R_{3}$ between bands gives a better spectral separation between components, and shows that SS resolves the same relative bands as FS. The concentration maps of the lipids retrieved using SS or FS are also very similar, as shown in the Figure S5.

It is also worth pointing out that in ref 13. we compared the outcome of the $\mathrm{FSC}^{3}$ analysis between measuring in the $\mathrm{CH}$ stretch vibrational range versus the fingerprint region, and found consistent results in terms of sensitivity to changes in lipid chemical profiles inside cells. This is because the reduction in spectral separation when analyzing the congested $\mathrm{CH}$-stretch region is compensated by the improved signal-to-noise ratio due to the higher CARS susceptibility in this vibrational range.

Screening for Drug-Induced Lipid Storage. Finally, we merged the ability to identify label-free markers of phospholipidosis via hyperspectral CARS (as shown in Figure 3) with the fast acquisition provided by Bessel beam excitation and 
sparse sampling $(45 \times$ faster than Gaussian beam illumination and full spectral acquisition for the settings used here), in order to screen a group of known and putative phospholipidosis inducers. Human HepG2 liver cells were treated with various combinations of compounds in a multiwell slide and then fixed (see Methods). First, two spectral components were identified in a similar way a described in the previous section about labelfree markers for phospholipidosis, using cells treated with Chloroquine as a known inducer for phospholipidosis ${ }^{30}$ and cells grown in medium supplemented with $O A$ as positive control for neutral lipid droplet formation. These spectra were then used as guides in the FSC ${ }^{3}$ analysis; images from all wells were analyzed as one set, to pull out common spectra and compare the concentration of these chemical components across the group (see Supporting Information). Figure 5a shows the spectra and concentration maps for the well B3 (chlorpromazine) for the two lipid components, which associate with phospholipidosis (red spectrum) and neutral lipid accumulation (green spectrum). The protein component which was retrieved in the same combined analysis is shown in the Figure S6.

We then tested if the high-content capability of the hyperspectral CARS analysis could be used to derive a set of predictors to train a support vector machine (SVM) discriminative algorithm and in turn classify the drug responses in terms of neutral lipid accumulation (steatosis) versus phospholipidosis in a completely label-free assay. From the pixel-by-pixel distribution of the protein and lipids concentration maps we extracted the mean value, the standard deviation, the skewness, the kurtosis, and the spatial correlation radius. Additionally, the mean values of the normalized spatial cross-correlations between the three components are included (see Supporting Information). This resulted in a total of 18 predictors per well/sample (typically $\sim 30$ cells were imaged and analyzed per well).

The calculated predictors are used to train a linear SVM model for two-class (binary) classification. To discriminate phospholipidosis (P) from steatosis (S) and control (C) cases, we performed three binary classifications: $P$ versus $S, P$ versus $\mathrm{C}$ and $\mathrm{S}$ versus $\mathrm{C}$. For the training sets, we use the predictors obtained from the images of the wells containing cells treated with Chloroquine (for P), medium supplemented with OA (for $\mathrm{S}$ ), and standard medium (for $\mathrm{C}$ ). Figure $5 \mathrm{c}$ gives a table of all the wells, and indicates the training sets labeled as stars (the sample unit is the entire image per well, i.e., 230 cells). Concentration maps for the wells used as training sets are given in Figure S8. The classification algorithm returns a hyperplane, and the normalized distance from it gives a measurement of the affinity with one of the two groups. To reduce the dimensionality of the predictor space, we trained each classifier with all possible subsets of the 18 predictors and selected the subset which was minimizing a figure of merit defined as detailed in the Supporting Information.

After training the classifiers, we calculated the normalized distance $d$ from the hyperplane for the wells not included in the training sets. The results of the classification analysis are shown for each binary classification in Figure 5c, and as an overview triangular-distance diagram in Figure $5 \mathrm{~b}$. We find that chlorpromazine and tamoxifen are classified as phospholipidosis inducers, consistent with previous studies. ${ }^{19,31}$ Notably, the combinations of chlorpromazine with $\mathrm{OA}$ and tamoxifen with $\mathrm{OA}$ are not classified as phospholipidosis inducer, suggesting that the addition of $\mathrm{OA}$ is able to affect the drug-induced phospholipidosis in these cases. Conversely, chloroquine with $\mathrm{OA}$ is still classified as phospholipidosis inducer. Compoundfree wells containing cells grown in standard media (with or without addition of the solvents used for the drugs) are all classified as control cases, as expected.

\section{CONCLUSIONS}

We have combined Bessel beam illumination with sparse sampling acquisition in hyperspectral CARS imaging for the first time, which enabled us to perform label-free high-content microscopy-based screening. For the setting used here, we show a 45-fold increase in speed (9-fold increase from the Bessel beam illumination compared to Gaussian beam excitation and 5-fold increase from sparse sampling compared to full spatial and spectral acquisition). This could be further increased by an even sparser sampling acquisition, eventually limited by the number of relevant spectral components and the signal-to-noise ratio. For example, in ref 27. we demonstrated sparse sampling with a reduction in the acquisition time by a factor of 25 , which, if combined with the Bessel beam illumination shown here, would lead to 225-fold increase in acquisition speed, making CARS imaging within a 384-well format (the most frequently used for high-throughput screening) achievable. The Bessel beam illumination shown here was optimized to provide a probe volume with an axial extension encompassing the full thickness of the $2 \mathrm{D}$ cell culture while retaining high confinement in-plane, thus enabling the sample's lateral organization to be probed at high resolution and averaging axially for fast acquisition. While further extension of the Bessel beam would only add unwanted volume in this case, it is practically possible, and might be desirable for thicker samples.

Hyperspectral CARS images were analyzed using our quantitative data analysis pipeline which retrieves Raman-like susceptibility spectra and factorizes them into water, proteins and lipid chemical components with associated concentration maps in vol/vol units. By examining cells subjected to known phospholipidosis inducers as well as cells in media supplemented with oleic acid, two chemical components were identified as label-free markers for phospholipidosis and steatosis (neutral lipid accumulation), in good agreement with state-of-the art fluorescence staining.

We screened 15 combinations of drugs and neutral lipids added to human HepG2 liver cells in a multiwell slide. From the pixel-by-pixel statistical distribution of the protein and lipids concentration maps and the normalized spatial crosscorrelations between the components within the cells, we extracted 18 predictors per well/sample. The calculated predictors were used to train three support vector machine binary discriminative algorithms which classified the drug responses in terms of steatosis versus phospholipidosis, control versus phospholipidosis, and control versus steatosis.

Although shown here for drug induced lipid storage within hepatic tissue, Bessel-beam hyperspectral CARS microscopy with sparse sampling is a general platform for label-free highcontent high-throughput quantitative chemical imaging. An area where it will be of specific interest, is for research into Nonalcoholic fatty liver disease, a condition that can affect up to $30 \%$ of the adult population, is found in $80 \%$ of obese and diabetic patients and can lead to liver cirrhosis if left unchecked. ${ }^{32,33}$ There are currently no approved pharmacological treatments for Nonalcoholic fatty liver disease, and recent small molecule screens have utilized triglyceride 
colorimetry assays to identify compounds that reduce lipid storage within primary hepatocytes, ${ }^{32}$ but can give no indication on the chemical composition of lipid storage during treatment. The high throughput hyperspectral analysis outlined within this paper would provide information as to how the lipid composition is changing within these cells, while also providing readout information on cell health indicators (such as phospholipidosis).

For cell models that are particularly difficult/impossible to stain within live assays, such as embryonic stem cells, mammalian eggs and embryos, this technique offers a solution to observe multiple chemical parameters in order to understand fundamental questions in cell biology through the label-free screening of phenotypes. It is also compatible and can run alongside fluorescent assays, allowing chemical profiling of cell populations while screening through classical fluorescent based imaging techniques, enabling multiparametric assessment with chemical sensitivity.

Notably, the application of this technology goes beyond its use in cell assays. The recent use of CARS in identifying atherosclerotic $^{34}$ and amyloid ${ }^{35}$ plaques opens up the prospect of implementing our method for quantitative chemical imaging/screening of tissue sections to identify regions of disease in an automated noninvasive label-free manner with content and throughput as yet unseen.

\section{ASSOCIATED CONTENT}

\section{S Supporting Information}

The Supporting Information is available free of charge on the ACS Publications website at DOI: 10.1021/acs.analchem.7b04039.

Additional text and figures on the Bessel beam generation and characterization, data analysis, and classification analysis (PDF)

\section{AUTHOR INFORMATION}

\section{Corresponding Author}

*E-mail: borrip@cf.ac.uk.

\section{ORCID}

Paola Borri: 0000-0002-7873-3314

\section{Author Contributions}

${ }^{\S}$ F.M. and I.P. contributed equally to this work.

\section{Notes}

The authors declare no competing financial interest.

Data availability information about the data created during this research, including how to access it, is available from Cardiff University data archive at http://doi.org/10.17035/d.2017. 0038287344 .

\section{ACKNOWLEDGMENTS}

This work was funded by the U.K. BBSRC Research Council (grant no. BB/H006575/1). Chloroquine and tamoxifen were gifts from Professor Arwyn Jones and Dr. Stephen Hiscox at the School of Pharmacy and Pharmaceutical Sciences at Cardiff University. Thanks to Kez Cleal for his help with Tissue Culture and the preparation of biological samples.

\section{REFERENCES}

(1) Boutros, M.; Heigwer, F.; Laufer, C. Cell 2015, 163, 1314-1325.
(2) Simpson, J. C.; Joggerst, B.; Laketa, V.; Verissimo, F.; Cetin, C.; Erfle, H.; Bexiga, M. G.; Singan, V. R.; Hériché, J.; Neumannet, B.; et al. Nat. Cell Biol. 2012, 14, 764-774.

(3) Bray, M.; Singh, S.; Han, H.; Davis, C.; Borgeson, B.; Hartland, C.; Kost-Alimova, M.; Gustafsdottir, S.; Gibson, C.; Carpenter, A. Nat. Protoc. 2016, 11, 1757-1774.

(4) Giacomini, K.; Krauss, R.; Roden, D.; Eichelbaum, M.; Hayden, M.; Nakamura, Y. Nature 2007, 446, 975-977.

(5) Siramshetty, V.; Nickel, J.; Omieczynski, C.; Gohlke, B.; Drwal, M.; Preissner, R. Nucleic Acids Res. 2016, 44, D1080-6.

(6) Schumacher, J.; Guo, G. Toxicol. Appl. Pharmacol. 2015, 289, 4047.

(7) Dash, A.; Figler, R.; Sanyal, A.; Wamhoff, B. Expert Opin. Drug Metab. Toxicol. 2017, 13, 193-204.

(8) Cheng, J.-X.; Xie, X. S. Science 2015, 350, aaa8870.

(9) Zumbusch, A.; Langbein, W.; Borri, P. Prog. Lipid Res. 2013, 52, 615-632.

(10) Evans, C. L.; Potma, E. O.; Puoris'haag, M.; Côté, D.; Lin, C. P.; Xie, X. S. Proc. Natl. Acad. Sci. U. S. A. 2005, 102, 16807-16812.

(11) Saar, B. G.; Freudiger, C. W.; Reichman, J.; Stanley, C. M.; Holtom, G. R.; Xie, X. S. Science 2010, 330, 1368-1370.

(12) Camp, C. H., Jr.; Lee, Y. J.; Heddleston, J. M.; Hartshorn, C. M.; Walker, A. R. H.; Rich, J. N.; Lathia, J. D.; Cicerone, M. T. Nat. Photonics 2014, 8, 627-634.

(13) Di Napoli, C.; Pope, I.; Masia, F.; Langbein, W.; Watson, P.; Borri, P. Anal. Chem. 2016, 88, 3677-3685.

(14) Masia, F.; Karuna, A.; Borri, P.; Langbein, W. J. Raman Spectrosc. 2015, 46, 727-734.

(15) Masia, F.; Glen, A.; Stephens, P.; Borri, P.; Langbein, W. Anal. Chem. 2013, 85, 10820-10828.

(16) Pope, I.; Langbein, W.; Watson, P.; Borri, P. Opt. Express 2013, 21, 7096-7106.

(17) Karuna, A.; Masia, F.; Borri, P.; Langbein, W. J. Raman Spectrosc. 2016, 47, 1167-1173.

(18) Camp, C. H., Jr.; Cicerone, M. T. Nat. Photonics 2015, 9, 295305.

(19) Shahane, S.; Huang, R.; Gerhold, D.; Baxa, U.; Austin, C.; Xia, M. J. Biomol. Screening 2014, 19, 66-76.

(20) Thériault, G.; Cottet, M.; Castonguay, A.; McCarthy, N.; De Koninck, Y. Front. Cell. Neurosci. 2014, 8, 139.

(21) Botcherby, E. J.; Juškaitis, R.; Wilson, T. Opt. Commun. 2006, 268, 253-260.

(22) Vuillemin, N.; Mahou, P.; Débarre, D.; Gacoin, T.; Tharaux, P.L.; Schanne-Klein, M.-C.; Supatto, W.; Beaurepaire, E. Sci. Rep. 2016, 6, 29863.

(23) Chen, X.; Zhang, C.; Lin, P.; Huang, K.-C.; Liang, J.; Tian, J.; Cheng, J.-X. Nat. Commun. 2017, 8, 15117.

(24) Heuke, S.; Zheng, J.; Akimov, D.; Heintzmann, R.; Schmitt, M.; Popp, J. Sci. Rep. 2015, 5, 10991.

(25) Heuke, S.; Legesse, F. B.; Akimov, D.; Hübner, U.; Dellith, I.; Schmitt, M.; Popp, J. J. Opt. Soc. Am. B 2015, 32, 1773-1779.

(26) Bandyopadhyay, S.; Klaunig, J.; Somani, P. Hepatology 1990, 12, $48-58$.

(27) Masia, F.; Borri, P.; Langbein, W. Opt. Express 2014, 22, 40214028.

(28) Di Napoli, C.; Pope, I.; Masia, F.; Watson, P.; Langbein, W.; Borri, P. Biomed. Opt. Express 2014, 5, 1378-1390.

(29) Di Napoli, C.; Masia, F.; Pope, I.; Otto, C.; Langbein, W.; Borri, P. J. Biophotonics 2014, 7, 68-76.

(30) Shayman, J.; Abe, A. Biochim. Biophys. Acta, Mol. Cell Biol. Lipids 2013, 1831, 602-611.

(31) Kasahara, T.; Tomita, K.; Murano, H.; Harada, T.; Tsubakimoto, K.; Ogihara, T.; Ohnishi, S.; Kakinuma, C. Toxicol. Sci. 2006, 90, 133-141.

(32) Yu, S.; Chen, E.; Sherwood, L.; Hull, M.; Woods, A.; Tremblay, M. ACS Chem. Biol. 2017, 12, 1937-1946.

(33) Chalasani, N.; Younossi, Z.; Lavine, J.; Diehl, A.; Brunt, E.; Cusi, K.; Charlton, M.; Sanyal, A. Hepatology 2012, 55, 2005-2023. 
(34) Lim, R. S.; Suhalim, J. L.; Miyazaki-Anzai, S.; Miyazaki, M.; Levi, M.; Potma, E. O.; Tromberg, B. J. J. Lipid Res. 2011, 52, 2177-2186. (35) Kiskis, J.; Fink, H.; Nyberg, L.; Thyr, J.; Li, J.-Y.; Enejder, A. Sci. Rep. 2015, 5, 13489. 